\title{
Eclipsing Binary Stars from Space
}

\author{
Hans Bruntt ${ }^{1}$ and John Southworth ${ }^{2}$ \\ ${ }^{1}$ School of Physics, University of Sydney, Australia \\ email: hans@bruntt.dk \\ ${ }^{2}$ Department of Physics, University of Warwick, UK \\ email: jkt@astro.keele.ac.uk
}

\begin{abstract}
We have begun a programme to obtain high-precision photometry of bright detached eclipsing binary (dEB) stars with the Wide-field InfraRed Explorer (wIRE) satellite (Bruntt \& Buzasi 2006). Due to the small aperture of WIRE, only stars brighter than $V=6$ can be observed. We are collecting data for about a dozen dEB targets and here we present preliminary results for three of them. We have chosen dEBs with primary components of B and early A type. One of our aims is to combine the information from the light curve analyses of the eclipses with asteroseismic information from analysis of the pulsation of the primary component.
\end{abstract}

Keywords. stars: binaries: eclipsing, stars: variables: other, stars: fundamental parameters

\section{Introduction}

The study of detached eclipsing binaries is of fundamental importance to stellar astronomy as a way of accurately measuring the parameters of normal stars from basic observational data (Andersen 1991). The masses and radii of detached eclipsing binary (dEB) stars can be measured to accuracies better than $1 \%$, and the effective temperatures and luminosities can be obtained from spectral analysis or the use of photometric calibrations. An important use of these data is in the calibration of theoretical models of stellar evolution, particularly if the component stars are of quite different mass or evolutionary stage (e.g. Andersen et al. 1991).

The important physical effects included in theoretical models, such as the extent of convective core overshooting and the efficiency of convective energy transport (mixing length), need to be constrained by observations to increase the predictive power of the models. This may be done by measuring increasingly accurate absolute properties of dEBs, which requires much improved observational data. It is arguably more difficult to significantly improve the quality and quantity of light curves, rather than radial velocity curves, because of the large amount of telescope time needed for each system. The KEPLER (Basri et al. 2005) and COROT (Baglin et al. 2001) space missions will help to solve this problem in the future by obtaining accurate and extensive light curves of a significant number of dEBs. The results presented here from the WIRE satellite give an indication of the level of accuracy that future space missions will provide.

\section{Three dEBs observed with WIRE}

We present preliminary results for three dEBs observed with WIRE: $\psi$ Centauri is a newly-discovered system and has a long orbital period, while AR Cassiopeiae and $\beta$ Aurigae are known systems with shallow eclipses. For these reasons observations with ground-based telescopes are difficult. $\psi$ Cen and AR Cas are particularly interesting systems since they have secondary components which have much smaller masses and radii than the primary stars so the eclipses are total. This allows us to determine the 


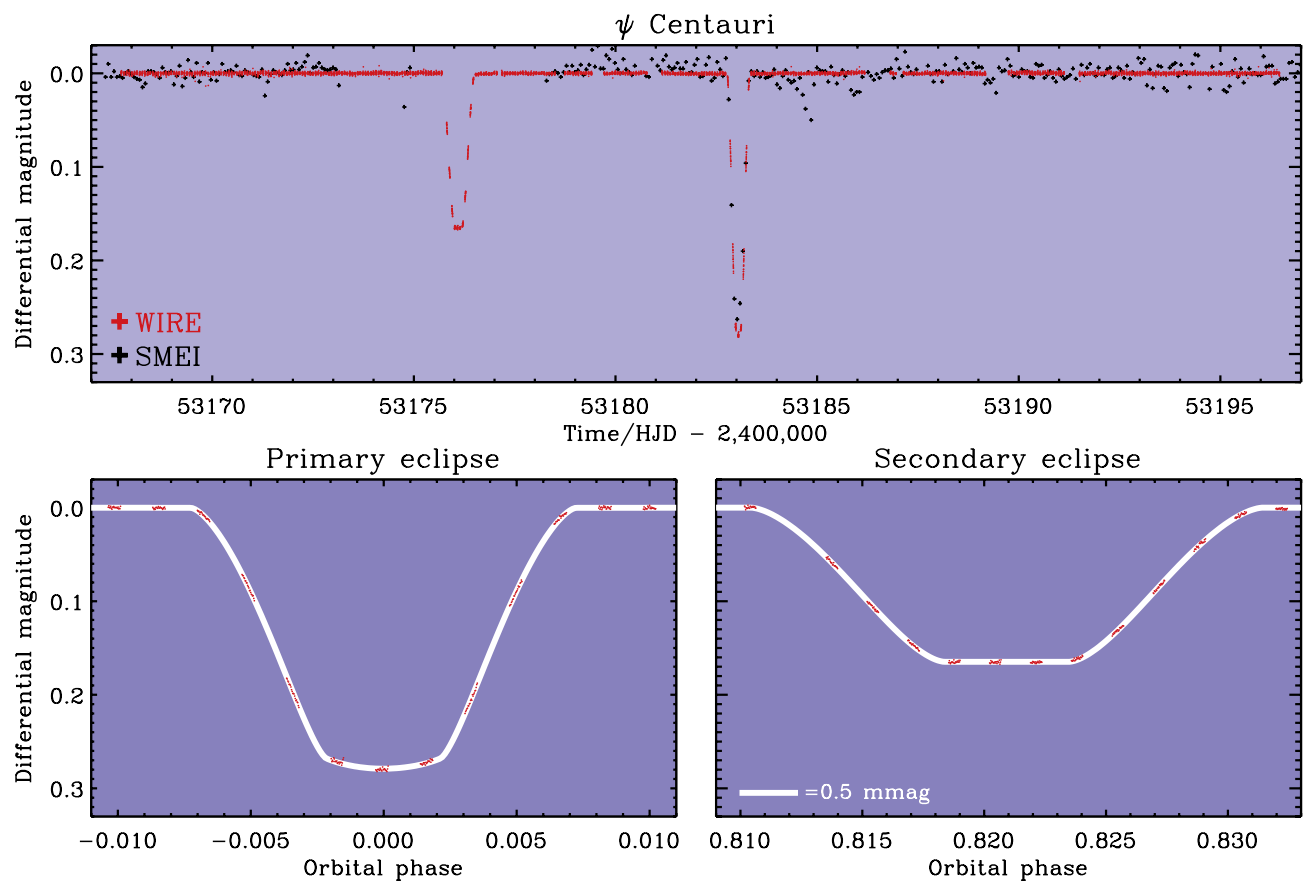

Figure 1. The light curve of $\psi$ Cen. In the top panel red points are from wIRE and black points are from SMEI. The bottom panels show the phased light curve around the primary and secondary eclipse.

radii with increased accuracy compared to $\beta$ Aur, which has partial eclipses. We are collecting new spectroscopic data for all systems, so we will be able to provide strict constraints on the predictions of theoretical models. The basic properties of the dEB systems we present here are given in Table 1 below and their light curves are shown in Figures 1-3.

\subsection{A new dEB: $\psi$ Centauri}

Serendipity led to the discovery that $\psi$ Cen is a dEB, as it was observed as a secondary target for another observing program. We used photometry with a time baseline of two years from the Solar Mass Ejection Imager (SMEI; Howard et al. 2006) on the Coriolis satellite to determine the period of $38.813 \mathrm{~d}$.

The light curve of $\psi$ Cen from WIRE is shown with red points in the top panel in Figure 1. The SMEI data that were collected at the same time as the discovery light curve from WIRE are shown with black points. The details of the primary and secondary eclipses are shown in the two bottom panels and the white curve is our best-fitting model

Table 1. Properties of the targets: Spectral type, magnitude, and period, time observed with WIRE, and precision per data point.

\begin{tabular}{l|ccc}
\hline & $\psi$ Cen & AR Cas & $\beta$ Aur \\
\hline Spectral type & A0 IV & B4 IV & A2 IV \\
$V$ & 4.0 & 4.9 & 1.9 \\
Period [d] & 38.813 & 6.066 & 3.960 \\
$\Delta T_{\text {obs }}$ & 28.7 & 26.4 & 20.9 \\
$\sigma_{\text {int }}[\mathrm{mmag}]$ & 1.0 & 1.1 & 0.1
\end{tabular}




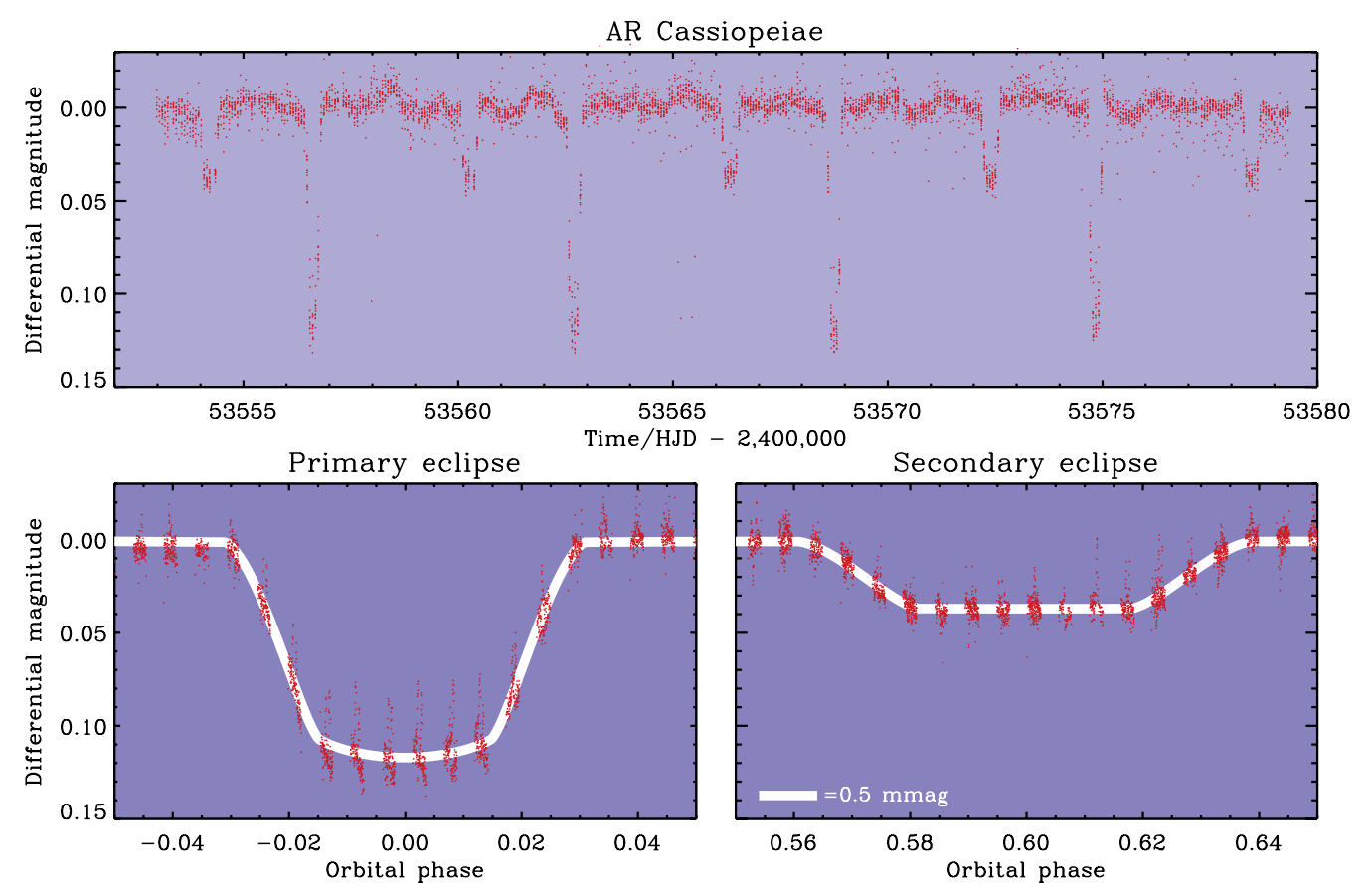

Figure 2. The light curve of AR Cas is shown in the top panel (every fifth data point is plotted). The bottom panels show the phased light curve around the primary and secondary eclipses. This is a preliminary data reduction and systematic offsets are seen in the light curve.

light curve. Bruntt et al. (2006) have made a detailed analysis of the light curve. From Monte Carlo simulations we find that the fractional radii of the two stars are determined to unprecedented accuracies of $0.1 \%$ and $0.2 \%$ (random errors). $\psi$ Cen is an interesting system since its brightest component is located in the region of the Hertzsprung-Russell diagram between the blue edge of the instability strip and the region of $g$-mode oscillations seen in slowly pulsating B stars (e.g., Pamyathnykh 1999). In the Fourier spectrum we detect two low-frequency modes that we interpret as global oscillation $g$-modes in the primary star.

\subsection{AR Cassiopeiae}

The light curve of AR Cas from WIRE is shown in the top panel in Figure 2 and the details of the primary and secondary eclipses are shown in the bottom panels. This is a preliminary result and systematic instrumental effects have not yet been fully removed. However, intrinsic variations in brightness are clearly present. Fourier analysis of the time series after subtracting the best-fitting light curve solution shows the presence of several pulsation modes with periods from 0.5 to 2.0 days. In addition, there is a brightness modulation at the rotational period of the primary component, which could be due to surface inhomogeneities in this star. The spectral type of AR Cas is B4 IV + A6 V and the brightness variations are possibly due to SPB-type pulsation in the primary star.

\section{3. $\beta$ Aurigae}

The light curve from WIRE of $\beta$ Aur is shown in Figure 3. The data have only been obtained very recently, and show 11 eclipses over a time span of 21 days. The phased light curve and a preliminary light curve model are shown in the bottom panels in Figure 3. 


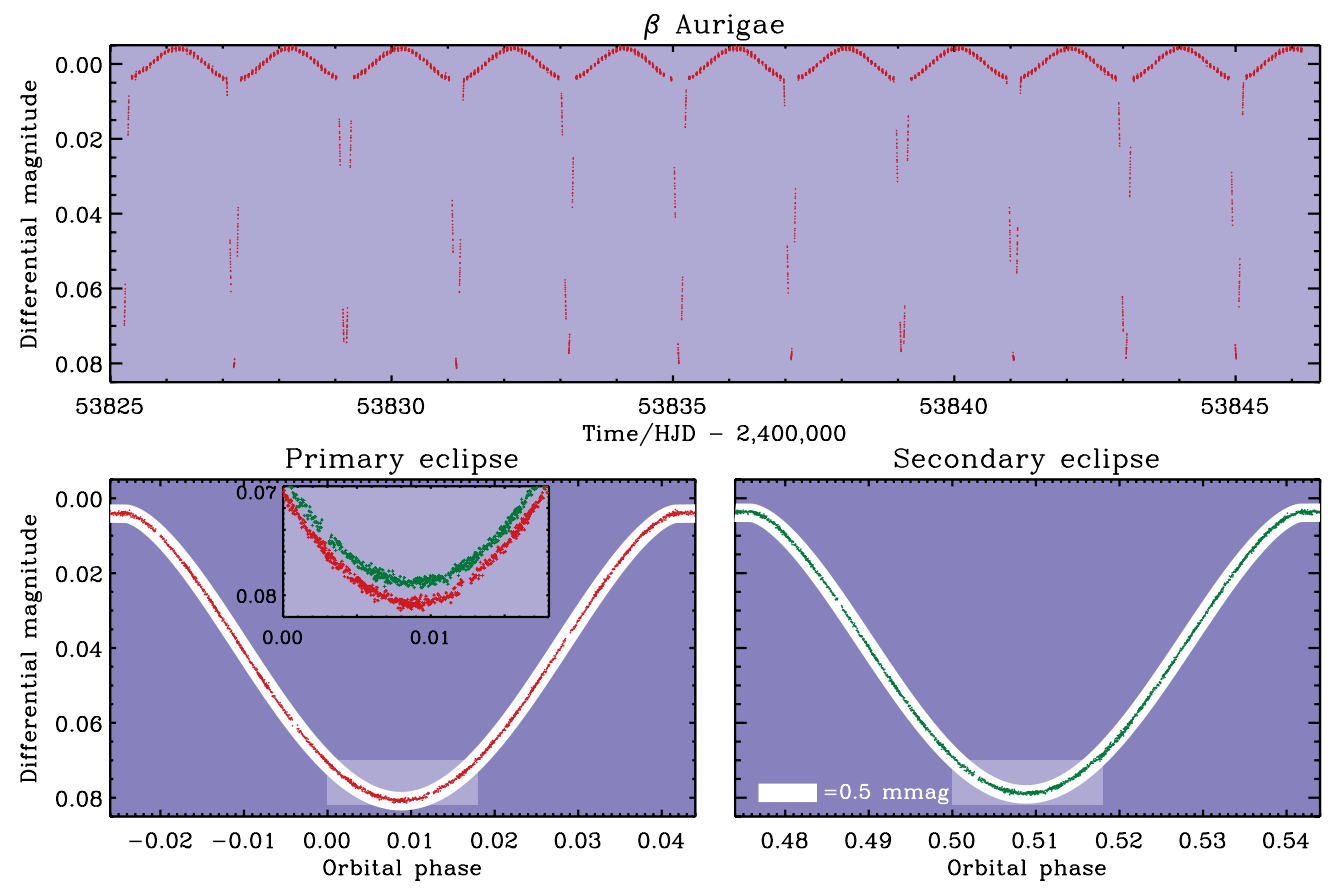

Figure 3. The light curve of $\beta$ Aur from WIRE is shown in the top panel (every fifth data point is plotted). The bottom panels show the phased light curve around the primary and secondary eclipses. The slight difference in the depths of the primary and secondary eclipses is seen in the the inset in the bottom left panel.

The inset in the bottom left panel shows the slightly different depths of the primary (red points) and secondary (green points) eclipses.

\section{Acknowledgements}

We thank Derek L. Buzasi (and the WIRE team), Jens Viggo Clausen, Guillermo Torres, Alan Penny (and the Coriolis \& SMei teams), Terry Moon, John Innis, Donald W. Kurtz, Thebe Medupe, Francois van Wyk, and Gerald Handler for providing valuable input and making this project possible.

\section{References}

Andersen, J. 1991, A\&AR, 3, 91

Andersen, J., Clausen, J.V., Nordstrom, B., Tomkin, J., \& Mayor, M. 1991, A\& A, 246, 99

Baglin, A., Auvergne, M., Catala, C., Michel, E., \& the Corot Team 2001, ESA SP-464: SOHO 10/GONG 2000 Workshop: Helio- and Asteroseismology at the Dawn of the Millennium, 10,395

Basri, G., Borucki, W.J., \& Koch, D. 2005, New Astronomy Review, 49, 478

Bruntt, H. \& Buzasi, D.L. 2006, Memorie della Societa Astronomica Italiana, 77, 278

Bruntt, H., Southworth, J., Torres, Penny, A.J., Clausen, J.V., \& Buzasi, D.L. 2006, Aש\&A, 456, 651

Howard, T.A., Webb, D.F., Tappin, S.J., Mizuno, D.R., \& Johnston, J.C. 2006, J. of Geophys. Res. (Space Physics), 111, A04105

Pamyatnykh, A.A. 1999, Acta Astronomica, 49, 119 